\title{
Serine/Threonine Protein Phosphatase 2B Catalytic Subunit Alpha Isoform
}

National Cancer Institute

\section{Source}

National Cancer Institute. Serine/Threonine Protein Phosphatase 2B Catalytic Subunit

Alpha Isoform. NCI Thesaurus. Code C38523.

Serine/threonine-protein phosphatase 2B catalytic subunit alpha isoform (521 aa, 59 $\mathrm{kDa}$ ) is encoded by the human PPP3CA gene. This protein is involved in calmodulin binding, protein dephosphorylation and signal transduction. 